\title{
Editorial
}

\section{Revolución 4.0 y las revistas científicas}

La tecnología presenta un escenario de cambios en el contexto editorial, producto de la aplicación de la inteligencia artificial (IA), las técnicas de análisis de grandes volúmenes de información (BigData), las telecomunicaciones de altas velocidades, la computación en nube y la alta automatización de procesos, avances que son considerados la cuarta revolución tecnológica o tecnologías 4.0.

Las tecnologías 4.0 sumadas a la amplia difusión del conocimiento, el número de investigadores en sectores públicos y privados, la elevada inversión en I+D+i (Investigación, Desarrollo e innovación); generan un diluvio de datos con la posibilidad de crear un amplio conjunto de métricas objetivas para le evaluación de la producción científica, las publicaciones, los autores y las temáticas entre otros aspectos que podrían ser de interés para evaluar el impacto de todos los actores en el sistema de producción de conocimiento.

En este escenario de gigantescos volúmenes de información, produciéndose a una tasa nunca vista por la humanidad, en un ambiente de creciente complejidad científica, donde producto de fenómenos sociales emergentes como los movimientos anti-ciencia que ponen en duda los resultados científicos; se genera un ambiente propicio para que se apueste por la IA como herramienta para automatizar muchos de los procesos necesarios para garantizar la calidad del producto editorial.

La integración de la IA y la automatización en el proceso editorial genera potenciales riesgos derivados de los sesgos inesperados que podrían emerger de integrar IA a un proceso que tradicionalmente ha sido subjetivo y basado principalmente en la relación entre pares y el editor de la publicación.

Es en los primeros filtros del proceso editorial, cuando el editor se relaciona de forma solitaria con la producción científica, donde se podrían presentar los principales riesgos, derivando en la imposibilidad de que algunos autores puedan difundir su producción por no cumplir con los parámetros de los algoritmos de apoyo, o que los autores terminen desarrollando toda su actividad en función de satisfacer al algoritmo por encima del mensaje que se desea transmitir, aspectos en los que se puede especular, ya que actualmente herramientas mucho más modestas como los correctores ortográficos y gramaticales ya tienen un impacto en el vocabulario y el estilo.

A pesar de los riesgos, es evidente las ventajas de las tecnologías 4.0, en la posible automatización de muchos de los procesos editoriales como, por ejemplo: la detección de plagio, la corrección de estilo, la evaluación de los autores, entre otros aspectos, que se pueden automatizar a partir de la aplicación de algoritmos apoyados en IA y la gran cantidad de información disponible.

Es relevante destacar que las nuevas herramientas producto de las tecnologías 4.0, beneficiarán a los autores y los editores a liberarlos de gran parte del trabajo administrativo, de edición, e interpretación de datos, entre otras tareas que se podrían automatizar, y que presentan una verdadera ventaja en el proceso editorial permitiendo a los autores enfocarse en la generación de nuevas ideas, el desarrollo creativo, entre otras tareas propias del autor, y a los editores, facilitar el proceso de comunicación y solución de controversias entre autores, pares evaluadores y el publico al que va dirigida la producción editorial.

Las tecnologías 4.0 que permiten la automatización de la producción de conocimiento, imponen un nuevo reto en el proceso editorial y de derechos de autor, consistente en cómo tratar los productos derivados de algoritmos de IA, pues actualmente es posible que un algoritmo de IA produzca un texto coherente e indistinguible de uno producido por un intelecto humano. 
En el contexto actual de la IA que permiten sintetizadores de texto a partir de algoritmos de interpretación del lenguaje natural, en donde los datos también son generados de forma automática con poca o ninguna intervención humana, no se tienen claridades sobre aspectos como la responsabilidad, los derechos morales y patrimoniales derivados del producto y los aspectos éticos inherentes derivados de los impactos del nuevo conocimiento.

Un posible escenario negativo, producto de estos cambios en donde el actor principal son las herramientas 4.0, es la dificultad que puede presentar la producción científica tradicional, frente a la que utiliza la IA para lograr el impacto y posicionamiento, además de la posible exclusión de autores, temáticas y fuentes; las cuales podrían ser discriminadas de forma automatizada en el proceso editorial.

Será entonces de gran importancia la existencia de publicaciones que den espacio a la controversia y a la relación humana entre pares, que apuesten por nuevas temáticas, nuevas fuentes que de otra manera no podrán incursionar en el ámbito Científico.

Julian Adolfo Ramírez Gutiérrez MSc. Electrónica y Telecomunicaciones Editor de sección Electrónica y Telecomunicaciones Universidad Surcolombiana 